ISSN 0103-5150

Fisioter. Mov., Curitiba, v. 24, n. 3, p. 429-436, jul./set. 2011 Licenciado sob uma Licença Creative Commons

\title{
Intervenção osteopática em idosos e o impacto na qualidade de vida
}

\author{
Osteopathic intervention in the elderly and its impact \\ on quality of life
}

\section{Luciana Cid Povoa $^{[a]}$, Fábio Kopp Vanuzzi ${ }^{[b]}$, Ana Paula Antunes Ferreira ${ }^{[c]}$, Arthur de Sá Ferreira ${ }^{[d]}$}

[a] Fisioterapeuta, osteopata D.O. MR(Br), Pós-Graduada em Geriatria e Gerontologia Interdisciplinar, Especialista em Reeducação Postural Global, professora assistente do Instituto Brasileiro de Osteopatia (IBO), Rio de Janeiro, RJ - Brasil, e-mail: lupovoa@yahoo.com.br

[b] Médico, Pós-Graduado em Geriatria, Pós-Graduado em Geriatria e Gerontologia Interdisciplinar, Pós-Graduado em Clínica de Dor e Cuidado Paliativo, Rio de Janeiro, RJ - Brasil.

[c] Fisioterapeuta, osteopata D.O. MR(Br), presidente do Registro Brasileiro dos Osteopatas, Especialista em Neurofisiologia, professora titular do Instituto Brasileiro de Osteopatia (IBO), Rio de Janeiro, RJ - Brasil.

[d] Fisioterapeuta, Doutor em Ciências em Engenharia Biomédica, professor adjunto do Centro Universitário Augusto Motta, Laboratório de Análise do Movimento Humano, Programa de Pós-Graduação em Ciências da Reabilitação, Centro Universitário Augusto Motta (UNISUAM), Rio de Janeiro, RJ - Brasil, e-mail: arthurde@profunisuam.com.br

\section{Resumo}

Introdução: 0 envelhecimento evidencia a necessidade do idoso do controle das doenças e do bem-estar físico, psíquico e social. Objetivo: Avaliar o impacto da intervenção osteopática na qualidade de vida de idosos. Materiais e métodos: Vinte e um indivíduos (72,1 \pm 4,7 anos, 18 mulheres) completaram este estudo. Foi aplicado o questionário de qualidade de vida WHOQOL-bref no primeiro e sexto atendimentos no intervalo de 48 [39; 66] dias. A avaliação e o tratamento das disfunções somáticas encontradas foram feitos em todas as sessões e as técnicas de domínio osteopático foram utilizadas. Os dados foram analisados por meio do teste de Wilcoxon com significância em $\mathrm{p}<0,05$. Resultados: Foi encontrada diferença significativa no domínio físico (pré-tratamento: 58,67 \pm 12,91\%; pós-tratamento: $64,64 \pm 13,75 \%$; p < 0,05). Não foram encontradas diferenças significativas nos domínios: psicológico (pré-tratamento: 63,10 \pm 15,38\%; pós-tratamento: 64,38 \pm 11,67\%; p = 0,846), relações sociais (pré-tratamento: $62,30 \pm 14,34 \%$; pós-tratamento: $62,50 \pm 12,82 \% ; p=1,000$ ) e meio ambiente (pré-tratamento: 52,08 \pm 11,91\%; pós-tratamento: 52,19 \pm $11,02 \% ; p=0,806)$. 0 nível de escolaridade apresentou associação significativa com a pontuação total do 
questionário antes ( $r=0,457 ; p=0,019)$ e após ( $r=0,380 ; p=0,049)$ a intervenção. Conclusão: A medicina osteopática, com sua ampla abordagem, interferiu positivamente no domínio físico da qualidade de vida. Outros domínios (meio ambiente, psicológico e relações pessoais) não foram modificados pela intervenção osteopática, contribuindo para a manutenção da qualidade de vida geral.

Palavras-chave: Medicina osteopática. Qualidade de vida. Idoso.

\section{Abstract}

Introduction: The aging process evidence the need to provide to the elderly either disease control and physical, psychological, and social well-being. Objective: To evaluate the osteopathic intervention and its impact in quality of life of aged people. Materials and methods: Twenty-one subjects ( $72.1 \pm 4.7$ years, 18 women) were enrolled in and completed this study. The WHOQOL-bref questionnaire was applied at first and sixth session with an average time interval of 48 [39; 66] days. At each session, screening tests and treatment were performed for somatic dysfunctions; treatment techniques were selected among osteopathic domain. Data from questionnaire were analyzed with Wilcoxon test with significance established at $p<0.05$. Results: Significant difference was found in the physical domain (before: $58.67 \pm 12.91 \%$; after: $64.64 \pm 13.75 \%$; $p<0.05$ ). No significant difference was found in psychological domain (before: $63.10 \pm 15.38 \%$; after: $64.38 \pm 11.67 \%$; $p=$ 0.846 ), social relations (before: $62.30 \pm 14.34 \%$; after: $62.50 \pm 12.82 \%$; $p=1.000$ ) and environment (before: $52.08 \pm 11.91 \%$; after: $52.19 \pm 11.02 \%$; $p=0.806)$. Educational level presented significant association with total scoring of the questionnaire before $(r=0.457 ; p=0.019)$ and after $(r=0,380 ; p=0,049)$ osteopathic intervention. Conclusion: Osteopathic medicine, in a broad approach, positively contributed to the physical domain of quality of life. Other domains (environmental, psychological, and social relations) were not modified by osteopathic intervention, resulting in unchanged overall quality of life.

Keywords: Osteopathic medicine. Quality of life. Elderly.

\section{Introdução}

0 termo envelhecimento é frequentemente empregado para descrever as mudanças morfofuncionais, ao longo da vida, que ocorrem após a maturação sexual e que, progressivamente, comprometem a capacidade de resposta dos indivíduos ao estresse ambiental e à manutenção da homeostasia (1). 0 envelhecimento da população é um dos maiores triunfos da humanidade e também um dos nossos grandes desafios (2). Poucos temas têm merecido tanta atenção como o envelhecimento e a incapacidade funcional comumente associada a ele (3). Em 2025, estima-se que existirá aproximadamente 1,2 bilhão de pessoas no mundo com mais de 60 anos, e o Brasil deverá ocupar o 6o lugar em número de idosos (2), com mais de 32 milhões de pessoas com idade igual ou superior a 60 anos (4). Algumas características marcantes da população que envelhece no Brasil são a pobreza e a baixa escolaridade (5).

As múltiplas facetas do processo do envelhecimento clamam para a necessidade de propiciar à pessoa idosa atenção abrangente à saúde, colocando em prática o que é preconizado pela Organização Mundial da Saúde: não somente o controle das doenças, mas o bem-estar físico, psíquico e social; em última análise, a melhora da qualidade de vida (6). Muitas funções fisiológicas declinam com a idade, incluindo velocidade de marcha, força muscular, quantidades de movimentos espontâneos, entre outros (7), o que acaba contribuindo para a alta prevalência de comorbidades nos idosos, redundando na busca por diversas especialidades da área, sobrecarregando o sistema de saúde. Tal sobrecarga, além de aumentar o custo da assistência, não representa necessariamente uma relação positiva de custo/efetividade, considerando que podem ocorrer iatrogenias importantes (como o uso inadequado de drogas) com consequências indesejáveis (6). A promoção de saúde e a profilaxia primária e secundária das doenças são as alternativas que apresentam o melhor custo-benefício para que se alcance a redução da morbidade nos idosos (8). A manutenção da qualidade de vida é provavelmente mais importante para o bem-estar 
do idoso do que a tentativa de tratar todas as suas doenças (9). Para atingir esse objetivo, a abordagem do cuidado do idoso deve ser holística e multidisciplinar, por meio de objetivos que busquem a manutenção da saúde e a melhora cinético-funcional. A compreensão do relacionamento entre a estrutura e a função (10), além da consideração das alterações homeostáticas ligadas à idade, é fundamental para o cuidado clínico geriátrico.

A medicina osteopática está baseada na interpretação do indivíduo como um ser único e integrado, mais do que a união de processos fisiológicos que ocorrem individualmente em diferentes sistemas (11, 12). Dessa forma, os osteopatas se concentram principalmente no funcionamento corporal, particularmente quando este se encontra desviado da fisiologia prevista (por exemplo, quando existe uma redução de movimento no próprio tecido e seus arredores, diminuindo a perfusão tecidual) (13). Observa-se então que os princípios e a prática osteopática se encaixam nos conceitos básicos do atendimento ao idoso (14). A abordagem global que a medicina osteopática propicia é essencial no cuidado geriátrico e envolve cuidados multidimensionais e interdisciplinares de avaliação e tratamento (15), considerando os aspectos médico, socioeconômico, psicológico e funcional do paciente. 0 relacionamento integrado da estrutura e função vem funcionando como alicerce da medicina osteopática e também pode ser considerado como a filosofia da medicina geriátrica, pois possui esse objetivo de manutenção da função (10).

Assim, as técnicas da medicina osteopática têm condições de contribuir para a promoção de um envelhecimento bem-sucedido e melhora na qualidade de vida (16). Entretanto, embora a qualidade de vida seja um assunto muito explorado em idosos $(2,8)$, no campo da medicina osteopática ainda não foi abordado em estudos prospectivos de intervenção; ainda faltam pesquisas nessa área que possam embasar essas afirmativas e os resultados obtidos clinicamente (16). Portanto, o objetivo deste estudo foi avaliar o impacto da intervenção osteopática na qualidade de vida do idoso.

\section{Materiais e métodos}

\section{Sujeitos}

Este trabalho foi um estudo prospectivo de intervenção realizado no Ambulatório de Osteopatia do
Hospital Estadual Anchieta (Caju, RJ). Vinte e sete indivíduos idosos foram avaliados para elegibilidade, neste estudo, e encaminhados para o Setor de Fisioterapia para o tratamento de desordens físicas variadas (Tabela 1). Não foram incluídos idosos que apresentaram déficit cognitivo que os impedisse de responder ao questionário ou que tivessem incapacidade de locomoção por meios próprios até o local de atendimento. Foram excluídos idosos que tenham permanecido mais de 15 dias sem atendimento (falta) e aqueles que abandonaram o tratamento sem completar os seis encontros. 0 idoso foi submetido ao questionário utilizado para triagem de acordo com os critérios do estudo. Após a triagem clínica e depois de serem informados sobre os objetivos e intervenções do estudo, os idosos assinaram o Termo de Consentimento Livre e Esclarecido. A pesquisa foi concluída com 21 idosos $(72,1 \pm 4,7$ anos, $18 \mathrm{mu}$ lheres; três abandonaram: um preferiu tratamento fisioterápico, um apresentou familiar doente e um relatou piora com o tratamento). Este trabalho seguiu as recomendações da Resolução 196/96 do Conselho Nacional de Saúde e foi aprovado pelo Comitê de Ética e Pesquisa em Humanos e Animais Institucional com Parecer número 160/2007.

\section{Procedimentos}

No primeiro dia de atendimento, foi aplicado o instrumento de aferição de qualidade de vida WHOQOLbref traduzido e validado no Brasil (17). A casuística se mostrou com um alto índice de analfabetismo, o que influencia na aplicação do questionário citado já que ele foi desenvolvido para ser autoaplicável. Para minimizar os efeitos do analfabetismo nas respostas do questionário, ele foi lido lentamente para os indivíduos analfabetos sem fornecer interpretações das questões ou sinônimos. Esse procedimento foi adotado atendendo à orientação fornecida antes do início da pesquisa pela equipe responsável pela tradução do WHOQOL-bref no Brasil.

A seguir, o sujeito foi avaliado segundo a abordagem osteopática, que consistiu em inspeção (observação de cicatrizes, vascularização, edemas, posição antálgica), testes de mobilidade tecidual, que visam a analisar a presença de alterações de mobilidade globais, regionais e locais, permitindo o diagnóstico osteopático, além da avaliação da amplitude de movimentos, reflexo, sensibilidade, força e marcha. 
Tabela 1 - Descrição da amostra

\begin{tabular}{|c|c|c|c|c|}
\hline $\mathbf{N}$ & $\begin{array}{l}\text { Idade } \\
\text { (anos) }\end{array}$ & Sexo & Distúrbio somático & Escolaridade \\
\hline 1 & 66 & $\mathrm{~F}$ & Lombalgia com irradiação & $1^{0}$ grau incompleto \\
\hline 2 & 75 & $\mathrm{~F}$ & Lombalgia sem irradiação, dor no ombro & Analfabeto \\
\hline 3 & 71 & $\mathrm{~F}$ & Lombalgia, dor na região anterior das coxas, hipoestesia nas mãos & 1ํgrau completo \\
\hline 4 & 66 & $\mathrm{~F}$ & Dor nos quadris, gonalgia & $1^{0}$ grau completo \\
\hline 5 & 73 & $\mathrm{~F}$ & Cervicalgia, cefaleia, dor no ombro & 10 grau incompleto \\
\hline 6 & 75 & $\mathrm{~F}$ & Gonalgia bilateral, lombalgia & 10 grau completo \\
\hline 7 & 69 & $\mathrm{~F}$ & Cervicalgia, dorsalgia, lombalgia, gonalgia & 10 grau incompleto \\
\hline 8 & 76 & $\mathrm{~F}$ & Cervicalgia, tonteira, dor temporal, dor nos quadris & $1^{0}$ grau completo \\
\hline 9 & 69 & M & Gonalgia, dor no ombro, dorsalgia, parestesia na mão & $1 \underline{0}$ grau completo \\
\hline 10 & 68 & $\mathrm{~F}$ & Gonalgia, dificuldade para agachar e andar, lombalgia, dor escapular, dor no punho & $1^{0}$ grau completo \\
\hline 11 & 77 & $\mathrm{~F}$ & Dor no ombro, "peso" nos quadris, "repuxos" na perna & Analfabeto \\
\hline 12 & 68 & $\mathrm{~F}$ & Lombalgia com irradiação, quedas & 10 grau incompleto \\
\hline 13 & 68 & $\mathrm{~F}$ & $\begin{array}{l}\text { Lombalgia com irradiação, contrações musculares do assoalho pélvico, } \\
\text { cervicalgia, dor nos dedos da mão, gonalgia bilateral }\end{array}$ & Analfabeto \\
\hline 14 & 73 & $\mathrm{~F}$ & Dor no ombro, dor no tórax & Analfabeto \\
\hline 15 & 74 & $\mathrm{~F}$ & Dor no ombro, dor costal, gonalgia, dor na sola dos pés & Analfabeto \\
\hline 16 & 68 & $\mathrm{~F}$ & $\begin{array}{l}\text { Cervicalgia, Iombalgia, dor no ombro, dificuldade para pegar peso e movimentar } \\
\text { MMSS, dificuldade de inspiração forçada }\end{array}$ & Analfabeto \\
\hline 17 & 72 & M & Dor no glúteo até 0 joelho & $1^{0}$ grau incompleto \\
\hline 18 & 78 & $\mathrm{~F}$ & Lombalgia com irradiação & Analfabeto \\
\hline 19 & 79 & M & Dor no glúteo até o joelho com parestesia & 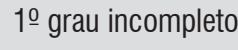 \\
\hline 20 & 67 & $\mathrm{~F}$ & Dor parietal, desconforto na coluna & $2^{0}$ grau incompleto \\
\hline 21 & 83 & $\mathrm{~F}$ & Lombalgia com irradiação, dor no ombro & 10 grau incompleto \\
\hline
\end{tabular}

Fonte: Dados da pesquisa.

Foi feito o tratamento necessário das disfunções somáticas encontradas, por meio da utilização de técnicas osteopáticas específicas (com exceção das técnicas de alta velocidade e baixa amplitude - que possuem como contraindicação a possibilidade de metástase óssea, osteopenia grave e história de fraturas patológicas) $(18,19)$.

A escolha das técnicas se baseou na indicação destas considerando o principal agente causador da desordem tecidual. Para as disfunções musculares - que possuem o vetor muscular como principal responsável pela disfunção de mobilidade -, a técnica escolhida foi a de energia muscular, que tenta neutralizar a ação desse componente de forma direta. Para as disfunções de origem fascial, as técnicas escolhidas foram de abordagem fascial, ou seja, de forma indireta (14). Também foram utilizadas as técnicas globais, como o tratamento geral osteopático (general osteopathic treatment - GOT), quando o diagnóstico é feito ao mesmo tempo em que o tratamento e se consegue abordar o indivíduo de maneira mais abrangente. As técnicas viscerais e cranianas seguiram a conduta de utilização segundo a sua necessidade, ou seja, quando na avaliação o principal elemento encontrado era de origem visceral, o tratamento era visceral, assim como o cranial. Em todas as abordagens de tratamento osteopático se faz necessária a reavaliação da disfunção, prática essa que nos permite aferir a eficácia da utilização das técnicas selecionadas.

Cada sessão teve a duração aproximada de uma hora e o idoso retornou para avaliação e tratamento osteopático por mais cinco sessões, com intervalo mediano de sete dias $[7 ; 14]$ entre as primeiras 
quatro sessões e intervalo mediano de 14 dias [7; 14] entre a quarta e a quinta sessão. A duração mediana do tratamento foi de $48[39 ; 66]$ dias. Na última sessão, após o atendimento osteopático, foi reaplicado o questionário WHOQOL-bref pelo mesmo avaliador que o aplicou ao início do estudo, seguindo os mesmos procedimentos de aplicação. Tanto a aplicação do questionário como a avaliação e o tratamento osteopáticos foram realizados pelo mesmo pesquisador (LCP), o qual seguiu as orientações de aplicação do WHOQOL-bref (17), além dos procedimentos de avaliação e tratamento osteopáticos de domínio da pesquisadora.

\section{Análise estatística}

Os dados referentes à queixa principal, escolaridade, duração do tratamento, idade e a pontuação do questionário WHOQOL-bref foram tabelados. As questões foram analisadas de acordo com os domínios: físico, psicológico, relações sociais e meio ambiente (17). A comparação entre os resultados do questionário antes e após o tratamento foi feita por meio do teste $t$ de Student pareado bicaudal. Considerando a observada frequência de analfabetos (aproximadamente 33\%), efetuou-se também uma análise por domínio e fase de tratamento (pré e póstratamento) entre os grupos de analfabetos e alfabetizados por meio do teste $t$ de Student não pareado bicaudal. A associação entre as variáveis estudadas foi testada com o coeficiente de correlação de Spearman. 0 nível de significância adotado foi de $\mathrm{p}<0,05$. Todas as análises foram executadas no programa SPSS ${ }^{\circledR} 17.0$ para Windows.

\section{Resultados}

Os resultados da comparação do questionário WHOQOL-bref por domínio antes e após o período de tratamento podem ser vistos na Tabela 2. Foi encontrada diferença significativa no domínio físico (pré-tratamento: $58,67 \pm 12,91 \%$; pós-tratamento: $64,64 \pm 13,75 \% ; p<0,05)$. Não foram encontradas diferenças significativas nos seguintes domínios: psicológico (pré-tratamento: $63,10 \pm 15,38 \%$; póstratamento: $64,38 \pm 11,67 \% ; p=0,846$ ), relações sociais (pré-tratamento: $62,30 \pm 14,34 \%$; pós-tratamento: $62,50 \pm 12,82 \% ; p=1,000$ ) e meio ambiente (pré-tratamento: 52,08 \pm 11,91\%; pós-tratamento: $52,19 \pm 11,02 \% ; p=0,806)$. Não foram encontradas diferenças significativas $(\mathrm{p}>0,05)$ na comparação de cada domínio (físico, psicológico, relações sociais e meio ambiente) em cada etapa da pesquisa (pré e pós-tratamento) entre os indivíduos analfabetos e alfabetizados.

O nível de escolaridade apresentou associação significativa com a pontuação total do questionário antes $(\mathrm{r}=0,457 ; \mathrm{p}=0,019)$ e após $(\mathrm{r}=0,380 ; \mathrm{p}=$ 0,049 ) a intervenção. Nenhuma outra comparação entre os domínios e as variáveis estudadas apresentou significância estatística.

Tabela 2 - Tabela de comparação do questionário de qualidade de vida por domínio, antes e após o período de tratamento

\begin{tabular}{lccc}
\hline Domínio & $\begin{array}{c}\text { Pré-tratamento } \\
\%\end{array}$ & $\begin{array}{c}\text { Pós-tratamento } \\
\%\end{array}$ & P-valor \\
\hline Físico & $58,67 \pm 12,91$ & $64,64 \pm 13,75$ & 0,024 \\
Psicológico & $63,10 \pm 15,38$ & $64,38 \pm 11,67$ & 0,846 \\
Relações sociais & $62,30 \pm 14,34$ & $62,50 \pm 12,82$ & 1,000 \\
Meio ambiente & $52,08 \pm 11,91$ & $52,19 \pm 11,02$ & 0,806 \\
\hline
\end{tabular}

Fonte: Dados da pesquisa.

\section{Discussão}

Este trabalho avaliou o impacto na qualidade de vida do tratamento osteopático em idosos. Os achados deste estudo sugerem que idosos submetidos à intervenção osteopática podem ser beneficiados sob o domínio físico. O benefício encontrado no domínio físico não se apresentou suficiente para melhorar a qualidade de vida geral. Como assinalado em outros estudos (20), parece que o aspecto saúde, quando perdido, representa um bom indicador de qualidade de vida negativa, porém mostra-se insuficiente para uma velhice bem-sucedida, uma vez que a qualidade de vida positiva equivale a uma pluralidade maior de categorias, como atividade, renda, vida social e relação com a família, categorias diferentes de sujeito para sujeito.

Os resultados da comparação do questionário de qualidade de vida, antes e após o período de tratamento, demonstraram melhora no domínio físico. 
As melhoras encontradas nesse domínio mostraram grande benefício para o indivíduo idoso, uma vez que os itens envolvidos mantêm relação importante na sensação de bem-estar, com menos impedimento na realização das atividades diárias e maior capacidade de locomoção, ou seja, mais independência. A qualidade de vida tem múltiplas dimensões, entre elas a saúde percebida e a capacidade funcional (21). Estudos epidemiológicos (22) sobre o envelhecimento vêm contribuindo substancialmente no entendimento da importância que a perda funcional tem no status de saúde do idoso. Para o idoso, quanto maiores a sua autonomia e independência, melhor será a sua qualidade de vida $(10,23)$. Para corrigir as desordens mecânicas do corpo, o osteopata utiliza manipulações terapêuticas. A normalização osteopática é suave e controlada. Isso pode ser direcionado para a mobilidade articular, muscular, fascial, visceral e cranial, objetivando intervir na circulação, na drenagem linfática e nos impulsos nervosos. 0 cerne da medicina osteopática é o reconhecimento da habilidade da autocura do organismo, com alguma ajuda externa, na maior parte das doenças (14).

A melhora funcional observada nos pacientes que foram submetidos ao tratamento osteopático pode ser explicada pela resolução de disfunções somáticas que esses pacientes apresentavam e que se relacionavam com a função prejudicada. Foi demonstrado (24) que o tratamento osteopático interfere de forma positiva em dores crônicas não específicas e que essa melhora poderia vir da própria manipulação osteopática, mas também de outros aspectos, como o aumento do arco de movimento proporcionado pela manobra ou o tempo de interação com o paciente. Não foi encontrado, na literatura consultada, nenhum trabalho semelhante, limitando a comparação de resultados entre outras pesquisas de mesma natureza. Além disso, os trabalhos (25-27) mais relevantes que relacionam o trabalho osteopático com indivíduos idosos vêm dos Estados Unidos, onde também existe o envolvimento medicamentoso na terapêutica desenvolvida, o que dificulta a comparação de resultados.

A escolha do número de sessões seguiu o protocolo utilizado no HEA, mas adequou o intervalo de atendimento entre uma sessão e outra para uma semana, de tal forma que cada paciente permaneceu na pesquisa por 48 [39; 66] dias. A opção pelo atendimento semanal levou em consideração a cronologia do trabalho. A literatura não apresenta nenhuma restrição com relação à frequência do tratamento proposto de seis semanas consecutivas (14). Tal variação ocorreu também por motivos externos à pesquisa, envolvendo segurança pública e recessos institucionais de atendimento. Por esses motivos, foi necessário flexibilizar o intervalo entre atendimentos de 7 a 14 dias para que os pacientes pudessem se manter sob atendimento. A variação no intervalo entre atendimento pode implicar diferentes graus de efeitos observados em resposta à intervenção terapêutica - os pacientes tratados com maior intervalo podem apresentar menos efeito em razão da falta de sobreposição de estímulos terapêuticos mais frequentes. Entretanto, considerando que o intervalo entre atendimentos foi distribuído entre pacientes e que a mediana de intervalo entre as sessões foi praticamente homogênea (com exceção da última sessão), optou-se por manter os pacientes que foram atendidos mesmo com intervalos maiores de até 14 dias.

Observou-se que o questionário, apesar de orientar que o entrevistado responda às perguntas com base nos acontecimentos das últimas duas semanas, sofre interferência do bem-estar do indivíduo no momento de resposta. Isso pode ser verificado por meio da análise da queixa principal com a avaliação final do questionário de qualidade de vida. Apesar de a maioria dos idosos ter ficado assintomática ou ter tido atenuação de seus sintomas ao fim do tratamento, não houve uma tradução literal desse fato com relação à melhoria da qualidade de vida geral.

Nos outros domínios (psicológico, relações sociais e meio ambiente) não houve melhora da qualidade de vida, porém supõe-se que esses resultados não tenham relação com intervenção osteopática. A pesquisa foi realizada na zona norte da cidade do Rio de Janeiro, que atende a uma população de baixa renda e escolaridade. Não foi encontrado nenhum questionário específico que avalie pessoas com baixa escolaridade; não há impossibilidade do uso desse questionário em pessoas com baixa escolaridade ou analfabetismo. Entretanto, ressalta-se que o analfabetismo não era um assunto pesquisado em países de primeiro mundo e que os países com analfabetos, em geral, têm pouca tradição em pesquisa com essa população. Na prática, o WHOQOLbref tem sido usado com analfabetos sendo administrado pelos entrevistadores. Mesmo seguindo as instruções dadas para a utilização do questionário, 
notou-se muita dificuldade de compreensão deste, 0 que pode ter influído nas respostas fornecidas. Graças ao mesmo fator descrito anteriormente, os pacientes idosos apresentaram muita dificuldade de compreensão e cooperação nas técnicas osteopáticas que necessitam da participação do paciente, como é o caso das técnicas fasciais de Sutherland e de energia muscular de Mitchell.

Ainda que a qualidade de vida seja "um conceito muito amplo que incorpora de uma maneira complexa a saúde física de uma pessoa, seu estado psicológico, seu nível de dependência, suas relações sociais, suas crenças e sua relação com características proeminentes no ambiente" (19), existe um instrumento utilizado e aceito mundialmente como forma de aferir quantitativamente a subjetividade desse assunto. Por esse motivo, o WHOQOL-bref foi o instrumento escolhido para verificar a eficiência do tratamento osteopático, no que diz respeito ao impacto que ele pode causar na qualidade de vida dos idosos. Foi utilizado o questionário, traduzido e validado no Brasil, em dois tempos (antes e após o tratamento osteopático). A inexistência de um questionário de qualidade de vida específico para a população idosa, pois o desenvolvimento do WHOQOL-OLD ainda se encontra em fase de construção (28), determinou a escolha do modelo em sua forma abreviada, porém, sem distinção de idade. A preferência do questionário abreviado levou em consideração o seu tamanho, que na versão integral (WHOQOL-100) é composto por 100 perguntas, enquanto a versão abreviada traz o total de 26 perguntas. Apesar de mais completo, um questionário mais amplo poderia trazer dificuldades operacionais, como, por exemplo, a dificuldade de compreensão do texto em função da baixa escolaridade encontrada nos idosos que participaram do estudo.

Está descrito que pacientes que buscam os setores de reabilitação para intervenção osteopática relatam qualidade de vida pior - não explicada pela idade - do que é geralmente reconhecido quando submetidos a abordagens diagnósticas da medicina convencional (29). Este estudo revelou, pela primeira vez, resultados positivos da intervenção osteopática na qualidade de vida (domínio físico) de idosos submetidos à intervenção osteopática. 0 tratamento osteopático é um recurso não invasivo, não medicamentoso e que pode ser utilizado no paciente idoso visando a ajustes que promovam melhora na qualidade de vida. Os achados deste estudo reforçam a hipótese de que a medicina osteopática tem condições de contribuir para a promoção de um envelhecimento bem-sucedido e melhora das condições físicas relacionadas à qualidade de vida.

\section{Conclusão}

Este trabalho mostrou que a medicina osteopática, com sua ampla abordagem, foi capaz de interferir positivamente no domínio físico, que faz parte da construção de uma qualidade de vida melhor. Outros domínios (meio ambiente, psicológico e relações pessoais) não foram modificados pela intervenção osteopática, contribuindo para a manutenção da qualidade de vida geral.

\section{Referências}

1. Jeckel-Neto EA, Cunha GL. Teorias biológicas do envelhecimento. In: Freitas E, Py L, Cançado FAX, Gorzoni M, editores. Tratado de geriatria e gerontologia. 2a ed. Rio de Janeiro: Guanabara Koogan; 2006. p. 14-22.

2. World Health Organization. Envelhecimento ativo: uma política de saúde. Brasília: Organização PanAmericana da Saúde; 2005.

3. Netto MP. 0 estudo da velhice: histórico, definição do campo e termos básicos. In: Freitas E, Py L, Cançado FAX, Gorzoni ML, editores. Tratado de geriatria e gerontologia. 2a ed. Rio de Janeiro: Guanabara Koogan; 2006. p. 2-12.

4. Netto MP. Epidemiologia do envelhecimento. In: Carvalho Filho ET, Netto MP, editores. Geriatria: fundamentos, clínica e terapêutica. 2a ed. São Paulo: Atheneu; 2005. p. 19-34.

5. Organização das Nações Unidas. Plan de acción internacional de Viena sobre el envejecimiento. Viena, Áustria; 1982.

6. Veras R. Envelhecimento humano: ações de promoção à saúde e prevenção de doenças. In: Freitas E, Py L, Cançado FAX, Gorzoni ML, editores. Tratado de geriatria e gerontologia. 2a ed. Rio de Janeiro: Guanabara Koogan; 2006. p. 140-6.

7. Cummings SR. The biology of aging. J Musculoskelet Neuronal Interact. 2007;7(4):340-1. 
8. Chaimowicz F. Saúde dos idosos brasileiros às vésperas do século XXI: problemas, projeções e alternativas. Rev Saúde Públ. 1997;31(2):184-200.

9. Lawton MP. Environment and other determinants of well-being in older people. Gerontologist. 1983;23(4): 349-57.

10. Cavalieri TA. Geriatrics. In: Ward RC, Jerome JA, Jones JM, Kappler RE, Kelso AF, Kuchera ML et al., editors. Foundations for osteopathic medicine. Philadelphia: Lippincot Williams \& Wilkins; 2003. p. 327-37.

11. Parsons J, Marcer N. What is osteopathy? Towards a definition. In: Parsons J, Marcer N, editores. Osteopathy: models for diagnosis, treatment and practice. London: Elsevier Churchill Livingstone; 2006. p. 3-14.

12. Digiovanna EL, Schiowitz S. Introduction to osteopathic medicine. In: Digiovanna EL, Schiowitz S, Dowling DJ, editors. An osteopathic approach to diagnosis and treatment. Philadelphia: J.B. Lippincott Company; 1991. p. 2-3.

13. Sammut E, Searle-Barnes P. Osteopathic concepts. In: Sammut E, Searle-Barnes P. Osteopathic diagnosis. London: Nelson Thornes; 1998. p. 3-23.

14. Parsons J, Marcer N. Treatment planning. In: Parsons J, Marcer N, editores. Osteopathy: models for diagnosis, treatment and practice. London: Elsevier Churchill Livingstone; 2006. p. 323-30.

15. Cavalieri TA. Managing pain in geriatric patients. J Am Osteopath Assoc. 2007;107(4):10-6.

16. Cavalieri TA. Clinical care for an aging population: aging successfully in the 21st century. J Am Osteopath Assoc. 2006;106(7):384-6.

17. Fleck MPA, Louzada S, Xavier M, Chachamovich E, Vieira G, Santos L, et al. Aplicação da versão em português do instrumento WHOQOL-bref. Rev Saúde Públ. 2000;34(2):178-83.

18. Lesho EM. An overview of osteopathic medicine. Arch Fam Med. 1999;8(6):477-84.

19. Schierhorn C. Complete care: elderly patients need gentle, individualistic, multidisciplinary approach. The D0. 2004:28-34.
20. Xavier FMF, Ferraz MPT, Marc N, Escosteguyc NU, Moriguchia EH. Elderly people's definition of quality of life. Rev Bras Psiquiatr. 2003;25(1):31-9.

21. Paschoal SMP. Qualidade de vida na velhice. In: Freitas E, Py L, Cançado FAX, Gorzoni ML, editores. Tratado de geriatria e gerontologia. 2a ed. Rio de Janeiro: Guanabara Koogan; 2006. p. 147-53.

22. Fried LP. Epidemiology of aging. Epidemiol Rev. 2000; 22(1):95-106.

23. Landini D. Idosos: qualidade de vida exige o resgate da participação social. Revista do INCOR. 2000: 6(62):6-17.

24. Licciardone JC, Stoll ST, Fulda KG, Russo DP, Siu J, Winn W, et al. Manipulative treatment for chronic low back pain: a randomized controlled trial. Spine. 2003;28(13):1355-62.

25. Cavalieri TA. Management of pain in older adults. J Am Osteopath Assoc. 2005;105(3):12-7.

26. Cavalieri TA. Pain management in the elderly. J Am Osteopath Assoc. 2002;102(9):481-5.

27. Kuchera ML. Osteopathic manipulative medicine considerations in patients with chronic pain. J Am Osteopath Assoc. 2005;4(9):529-36.

28. Fleck MPA, Chachamovich E, Trentini CM. Projeto WHOQOL-OLD: método e resultados de grupos focais no Brasil. Rev Saúde Pública. 2003;37(6):793-9.

29. Licciardone JC, Gamber RG, Russo DP. Quality of life in referred patients presenting to a specialty clinic for osteopathic manipulative treatment. J Am Osteopath Assoc. 2002;102(3):151-5.

Recebido: 10/01/2011 Received: 01/10/2011

Aprovado: 14/04/2011 Approved: 04/14/2011 